\title{
Morbilidad extrema (near-miss) en obstetricia. Revisión de la literatura
}

\section{Severe morbidity (near-miss) in obstetrics. Literature review}

\author{
Dr. Armando Alberto Moreno-Santillán, ${ }^{*}$ Dr. Carlos Gabriel Briones-Vega, ${ }^{\ddagger}$ \\ Dr. Manuel Antonio Díaz de León-Ponce, ${ }^{\S}$ Dr. Enrique Gómez Bravo-Topete,॥ \\ Dra. Leidy Marcela Martínez-Adame, " Dr. Martín Rodríguez-Roldán, ${ }^{* *}$ \\ Dr. Eduardo Sandoval-Rodríguez, ${ }^{\ddagger}$ Dr. Jesús Carlos Briones-Garduño§§
}

RESUMEN. Objetivo: Presentar la evidencia científica más reciente referente a la definición, diagnóstico y consecuencias de morbilidad extrema en obstetricia (MEO). Metodología de búsqueda: Búsqueda en PubMed, The Cochrane Library, OVID, Science Direct, Google Académico, Artemisa, LILACS e Imbiomed de artículos publicados en inglés y español entre los años 2005 a 2018 con las siguientes palabras clave: severe maternal morbidity, near miss morbidity, severe acute maternal morbidity, obstetric near miss and maternal near miss. Se excluyeron estudios cualitativos. Resultados: La MEO (o near miss) se refiere a cualquier complicación aguda que puede presentarse en el embarazo, labor o hasta seis semanas después de haber concluido el embarazo, estas complicaciones ponen en riesgo la vida de la madre, pero no resulta en su muerte. Los indicadores de MEO fueron desarrollados con base a la incidencia y presencia de trastornos hemorrágicos, hipertensivos y otras alteraciones sistémicas y condiciones maternas graves que pueden tener un impacto en la salud de la madre. En la actualidad, se sugiere que el estudio de los indicadores de MEO son de más utilidad que los de muerte materna. Conclusiones: El ejercicio de la práctica médica con base en la mejor evidencia científica, el estudio y la mejora de la calidad de la práctica clínica, de la planificación familiar, la asesoría prenatal y los programas hospitalarios son estrategias que permitirán ayudar a disminuir los casos de MEO.

ABSTRACT. Objective: To provide the most recent scientific evidence about definitions, diagnosis and consequences of severe maternal morbidity (SMM). Research methodology: We searched databases in PubMed, The Cochrane Library, OVID, Science Direct, Google Scholar, Artemisa, LILACS and Imbiomed from 2005 to 2018 with the following keywords: severe maternal morbidity, near miss morbidity, severe acute maternal morbidity, obstetric near miss and maternal near miss. The search was restricted to articles written in the English and Spanish language and published from 2005 to 2018. Qualitative studies were excluded. Results: SMM or maternal near miss event refers to any acute obstetric complication that immediately threatens a woman's survival but does not result in her death either by chance or because of hospital care she receives during pregnancy, labor or within six weeks of termination of pregnancy. The indicators for SMM were developed and based on the incidence and presence of hemorrhagic disorders, hypertensive disorders, other systemic disorders and severe maternal conditions which could have an impact on maternal health. Nowadays it is suggested that the study of indicators for SMM is a more useful indicator of obstetric care than mortality. Conclusions: Use of best evidence-based practices, studying and improving the effectiveness and quality of clinical practice, family planning, prenatal check-up, and hospital obstetric care programs are strategies that could help to reduce cases of SMM.
Solicitud de sobretiros:

Dr. Armando Alberto

Moreno-Santillán

Tlacotalpan Núm. 59,

Torre Diamante,

consultorio 410,

Col. Roma Sur, 06760,

Alcaldía Cuauhtémoc,

Ciudad de México.

E-mail: armorno@gmail.com
Palabras clave:

Morbilidad extrema, embarazo, puerperio.

Keywords:

pregnancy, puerperium.
Severe maternal morbidity,
Recibido para publicación:

25-07-2018

Aceptado para publicación:

10-10-2018
* Médico Gineco-obstetra adscrito a la Unidad Médica de Alta Especialidad, Hospital de Ginecología y Obstetricia No. 4 «Luis Castelazo Ayala». Instituto Mexicano del Seguro Social, México. Profesor de Postgrado de la Facultad de Medicina de la Universidad Nacional Autónoma de México.

‡ Médico Gineco-obstetra Materno-Fetal, Jefe de la Unidad de Perinatología del Instituto de Genética e Infertilidad, México.

$\S$ Médico Intensivista y Nefrólogo. Académico Titular de la Academia Nacional de Medicina. Académico Emérito de la Academia Mexicana de Cirugía.

॥ Médico Gineco-obstetra, Académico Titular de la Academia Mexicana de Cirugía, Titular de la Delegación Poniente IMSS del Estado de México, Exsecretario de Salud y Profesor de la Facultad de Medicina de la Universidad Autónoma del Estado de México.

" Médico Gineco-obstetra adscrito al Hospital Ángeles Metropolitano.

** Médico Gineco-obstetra, Director del Hospital de GinecoObstetricia 221, IMSS. Toluca, Estado de México.

\# Médico Gineco-obstetra, Subdirector del Hospital de GinecoObstetricia 221, IMSS. Toluca, Estado de México.

$\S \S$ Médico Gineco-obstetra e Intensivista. Académico de la Academia Nacional de Medicina y Academia Mexicana de Cirugía. Jefe de Servicios de Ginecología y Obstetricia del Hospital General de México «Dr. Eduardo Liceaga». Profesor Titular de la Especialidad de Medicina Crítica en Obstetricia Universidad Nacional Autónoma de México. 


\section{INTRODUCCIÓN}

D urante el ejercicio de la atención obstétrica, la mayoría de las mujeres tiene una atención adecuada y libre de efectos adversos, algunas mujeres mueren y otras tienen alto riesgo de muerte, pero sobreviven ${ }^{(1)}$. Del estudio de este último grupo de mujeres surge el concepto anglosajón de near-miss, y del cual derivan las traducciones al castellano de «morbilidad extrema», «complicaciones maternas agudas graves» 0 «casi pérdida» $(2,3)$.

El término de near-miss fue propuesto por Stones en 1991, y hace referencia a un evento no planeado que no resulta en la muerte o lesión, pero que pudo haber sido, es decir, un evento que puso en riesgo la vida de la paciente, sin causarle la muerte ${ }^{(4)}$. El concepto de near-miss o morbilidad extrema en obstetricia (MEO) se entiende como aquella mujer que casi muere pero sobrevive a una complicación severa del embarazo, parto o puerperio (hasta el día 42 después de la conclusión del mismo). La importancia de su estudio radica en que al ser situaciones que no causan una repercusión negativa en la vida de la paciente (y que por lo general no son documentadas o reportadas) puede propiciar que el mismo evento se presente en el futuro con consecuencias funestas ${ }^{(5)}$.

\section{EPIDEMIOLOGÍA}

Se estima que por cada muerte materna existen 100 casos de MEO, por lo que, dada esta alta incidencia, estos eventos deben ser considerados como un importante problema de salud pública ${ }^{(6-8)}$. A nivel mundial se ha reportado una tasa de MEO del 4.8 por cada 1,000 nacimientos, siendo las causas más comunes la hemorragia postparto y la sepsis ${ }^{(9,10)}$. En los Estados Unidos de América se estima que existen 50,000 casos al año, lo que equivale al $2 \%$ de todos los nacimientos ${ }^{(11)}$. Los países del Continente Americano con más casos de MEO, de acuerdo con la Organización Mundial de la Salud (OMS), son Brasil, México y $\mathrm{Cuba}^{(12)}$. En México, según un estudio realizado en Oaxaca en 2010, la prevalencia de la morbilidad obstétrica extrema fue de $2.1 \%$, encontrando que las comorbilidades más frecuentes fueron eclampsia, insuficiencia hepática y preeclampsia $^{(13)}$.

\section{DIAGNÓSTICO}

Inicialmente la medición de los eventos de MEO fue difícil debido a la falta de criterios unificados para su diagnóstico y seguimiento, por lo que en 2009 la OMS reunió a un grupo de expertos en el área de la morbilidad materna y propusieron una serie de criterios estandarizados y definiciones que pueden ser empleadas por las unidades hospitalarias de cualquier nivel para identificar los casos de MEO (Tabla 1) ${ }^{(5)}$.
Los criterios diagnósticos de MEO de la OMS han sido empleados mundialmente para el estudio e implementación de proyectos de mejora en la calidad de atención hospitalaria; sin embargo, su aplicación en países en vías de desarrollo ha sido cuestionada, debido a que muchos hospitales no cuentan con todos los estudios de laboratorio que se incluyen en dichos criterios ${ }^{(14)}$.

En los últimos años dos grupos europeos EURO-PERISTAT y EPIMOMS propusieron una herramienta con 17 indicadores, elaborada específicamente para su uso en países desarrollados. También en Estados Unidos, la CDC publicó una lista de 18 indicadores, basados en el CIE-10, para facilitar la identificación de los eventos de $\mathrm{MEO}^{(15-17)}$.

Organizaciones como el Colegio Americano de Ginecología y Obstetricia (ACOG) y la Sociedad de Medicina Materno Fetal (SMFM) recomiendan el uso de dos criterios de tamizaje: transfusión de cuatro o más paquetes globulares y la admisión, durante el embarazo o el puerperio, a la Unidad de Cuidados Intensivos (UCI) ${ }^{(18)}$. Si la paciente cuenta con uno o dos de estos criterios, se deberá de revisar su caso para confirmar la MEO. Diversos estudios han demostrado que el empleo de estos criterios tienen una alta sensibilidad y especificidad para identificar mujeres con $\mathrm{MEO}^{(19-21)}$.

Tabla 1: Criterios diagnósticos de morbilidad extrema en obstetricia, según la $\mathrm{OMS}^{(5)}$.

Criterios clínicos

Cianosis aguda

Dificultad respiratoria

Frecuencia respiratoria $>400<6$ por minuto

Estado de choque

Oliguria refractaria a la reanimación

Coagulopatía

Pérdida de la conciencia por más de 12 horas

Pérdida de la conciencia con ausencia de pulso o latido cardíaco

Convulsiones incontrolables

Déficit neurológico de origen cerebrovascular por más de 24 horas

Ictericia asociada a preeclampsia

Criterios de laboratorio

Saturación de oxígeno $<90 \%$ por más de 60 minutos

$\mathrm{PaO}_{2} / \mathrm{FiO}_{2}<200 \mathrm{mmHg}$

Creatinina sérica $\geq 3.5 \mathrm{mg} / \mathrm{dL}$

Bilirubina $\geq 6 \mathrm{mg} / \mathrm{dL}$

$\mathrm{pH}<7.1$

Lactato $>5$

Trombocitopenia aguda $(<50,000)$

Glucosuria y cetonuria asociadas con pérdida de la conciencia

Criterios terapéuticos

Uso de fármacos vasoactivos

Histerectomía debida a hemorragia o infección

Transfusión de más de cinco paquetes globulares

Intubación y ventilación asistida por más de 60 minutos (no relacionado

al procedimiento anestésico)

Diálisis por insuficiencia renal aguda

Paro cardiorrespiratorio 


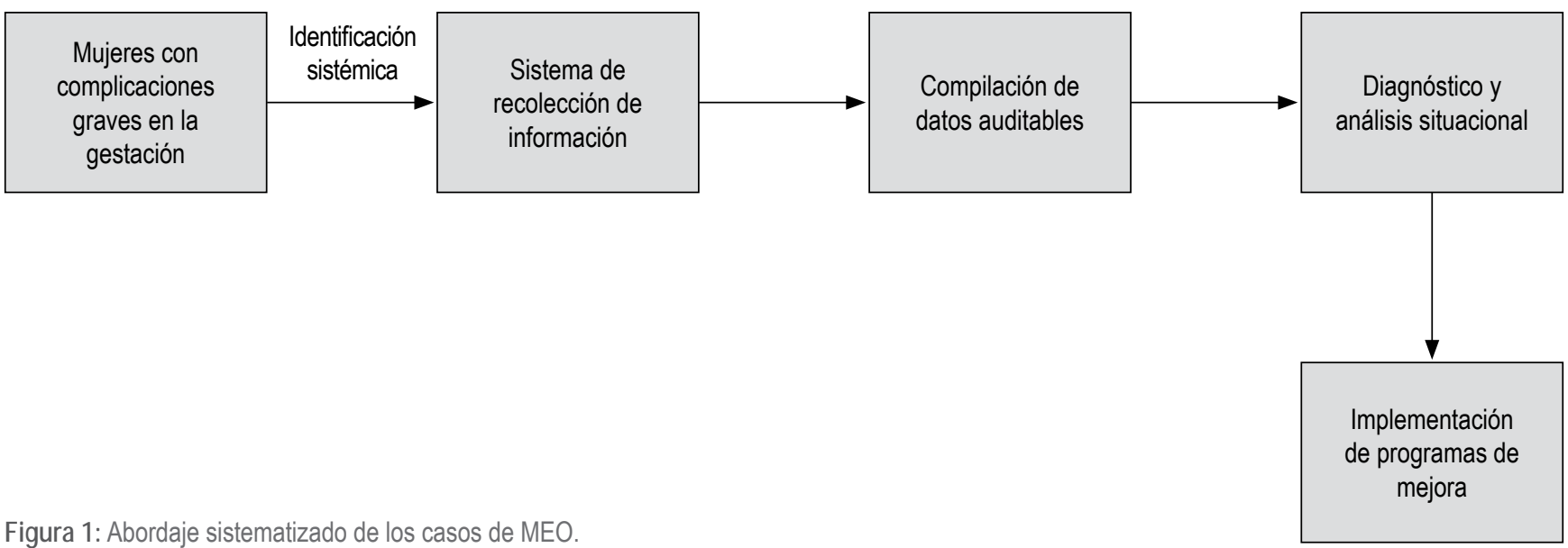

En la actualidad, la evaluación de los eventos de MEO es considerada como un importante indicador de la calidad hospitalaria, el cual anteriormente se evaluaba mediante la estimación de la mortalidad materna; sin embargo, al considerar únicamente la mortalidad se pasaba por alto la morbilidad y los eventos que ponían en riesgo la vida de la paciente sin causar la muerte, por lo que las acciones correctivas resultaban insuficientes ${ }^{(3)}$.

\section{ANÁLISIS DE LOS CASOS DE MEO}

La OMS ha recomendado que en cada unidad hospitalaria se implemente un sistema de vigilancia y evaluación de la calidad de los servicios de salud en obstetricia, con la finalidad de determinar la frecuencia de las complicaciones maternas, los casos de MEO y de muerte materna, el desempeño del personal de salud y evaluar la frecuencia de las intervenciones clave para la prevención de complicaciones graves relacionadas con el nacimiento, lo que finalmente promoverá la implementación de programas de mejora en la calidad de la atención materna ${ }^{(1,22)}$.

El estudio debe iniciar con el análisis de cada caso particular en cada centro hospitalario, para después integrar un diagnóstico distrital que pueda ser escalado a nivel del Sistema de Salud Nacional ${ }^{(1)}$. Se deben de identificar los casos basados en un programa que permita clasificar los tipos y características de los escenarios de MEO. La obtención de las variables debe de provenir de la revisión de los expedientes clínicos de cada paciente, tomando en cuenta las complicaciones relacionadas con el embarazo, las intervenciones críticas realizadas y la admisión a la Unidad de Cuidados Intensivos (UCI) ${ }^{(1)}$. Por último, los resultados obtenidos deberán clasificarse en patrones causales de morbilidad o mortalidad materna, fortalezas y debilidades del sistema de referencia y el uso de intervenciones clínicas. La publicación de los resultados deberá ser motivo de la implementación de programas y políticas hospitalarias que le permitan al personal de salud y a la sociedad civil mejorar la calidad de los cuidados a la mujer embarazada ${ }^{(1)}$.

La OMS recomienda sistematizar el estudios de estos casos mediante la aplicación de tres pasos:

1. Evaluación inicial o basal

2. Análisis situacional.

3. Intervenciones de mejora.

Este abordaje sistematizado puede realizarse en unidades hospitalarias, coordinaciones distritales o a nivel del sistema de salud nacional (Figura 1).

\section{CONSECUENCIAS PERINATALES DE LA MEO}

La MEO no sólo pone en riesgo la vida de la madre, pues el feto o el recién nacido también puede padecer las consecuencias de la morbilidad extrema de la madre, como aumento del riesgo de óbito, ingreso a la Unidad de Cuidados Intensivos Neonatales, nacimiento pretérmino y restricción del crecimiento intrauterino. Estos hallazgos refuerzan la importancia de realizar acciones preventivas encaminadas a disminuir la MEO, pues los beneficios potenciales son para las madres y sus hijos ${ }^{(23,24)}$.

\section{CONCLUSIONES}

A pesar de los esfuerzos mundiales para disminuir la mortalidad materna, ésta continúa siendo inaceptablemente elevada, sobre todo en países en vías de desarrollo. Aunado a la preocupación por las cifras de muerte materna, es evidente que existe un marcado incremento en los casos de MEO, aún en países desarrollados, con las consecuencias maternas, perinatales y sociales que esto conlleva.

Para poder lograr una reducción de la morbilidad y mortalidad materna es necesario implementar programas que 
permitan identificar todos los casos y conocer los factores de riesgo que se relacionan con los eventos de MEO, para así poder realizar intervenciones encaminadas a su prevención, control y erradicación. Por tanto, es importante recalcar que el mejorar la efectividad y calidad de los programas de planificación familiar, control prenatal y atención obstétrica hospitalaria son medidas indispensables para reducir los casos de MEO. Finalmente, se debe tomar en cuenta que no todos los casos que cumplan con criterios diagnósticos representarán un caso de MEO, pues en algunas ocasiones la morbilidad reflejará la causa subyacente e inevitable de la evolución natural de la patología propia de la gestación.

\section{REFERENCIAS}

1. World Health Organization. Evaluating the quality of care for severe pregnancy complications. The WHO near-miss approach for maternal health. Switzerland: 2011. Disponible en: http://www.who.int/about/ licensing/copyright_form/en/index.html.

2. Franco YCE, Hernández PJA. Monitoreo de morbilidad materna extrema (near miss) como compromiso internacional para complementar la calidad de la atención en salud materna. Perinatol Reprod Hum. 2016;30:31-38.

3. Galindo MN, Roig CN, Moreno CA, Gurrea SM, Alberola CV, et al. Near-miss o casi pérdida en un hospital de referencia. Prog Obstet Ginecol. 2010;53:399-402.

4. Stones W, Lim W, Al-Azzawi F, Kelly M. An investigation of maternal morbidity with identification of life-threatening 'near miss' episodes. Health Trends. 1991;23:13-15.

5. Say L, Souza JP, Pattinson RC; WHO working group on Maternal Mortality and Morbidity classifications. Maternal near miss-towards a standard tool for monitoring quality of maternal health care. Best Pract Res Clin Obstet Gynaecol. 2009;23:287-296.

6. Costa OL, Ribeiro CA. Maternal near miss in the intensive care unit: clinical and epidemiological aspects. Rev Bras Ter Intensiva. 2015;27:220-222.

7. Penney G, Brace V. Near miss audit in obstetrics. Curr Opin Obstet Gynecol. 2007;19:145-150.

8. Kaye DK, Kakaire O, Osinde MO. Systematic review of the magnitude and case fatality ratio for severe maternal morbidity in sub-Saharan Africa between 1995 and 2010. BMC Pregnancy Childbirth. 2011;11:65.

9. van Roosmalen J, Zwart J. Severe acute maternal morbidity in high-income countries. Best Pract Res Clin Obstet Gynaecol. 2009;23(3):297-304.

10. Jayaratnam S, Burton A, Connan KF, de Costa C. Maternal 'near miss' at Royal Darwin Hospital: an analysis of severe maternal morbidity at an Australian regional tertiary maternity unit. Aust N Z J Obstet Gynaecol. 2016;56:381-386.

11. Kilpatrick SJ, Abreo A, Gould J, Greene N, Main EK. Confirmed severe maternal morbidity is associated with high rate of preterm delivery. Am J Obstet Gynecol. 2016;215:233.e1-7.

12. Souza JP, Cecatti JG, Faundes A, Morais SS, Villar J, Carroli G, et al. Maternal near miss and maternal death in the World Health Organization's 2005 global survey on maternal and perinatal health. Bull World Health Organ. 2010;88:113-119.
13. Calvo-Aguilar O, Morales-García VE, Fabián-Fabián J. Morbilidad materna extrema en el Hospital General Dr. Aurelio Valdivieso, Servicios de Salud de Oaxaca. Ginecol Obstet Mex. 2010;78:660-668.

14. Geller SE, Koch AR, Garland CE, MacDonald EJ, Storey F, Lawton B. A global view of severe maternal morbidity: moving beyond maternal mortality. Reprod Health. 2018;15:98.

15. Deneux-Tharaux C, Bouvier-Colle MH, EPIMOMS Study Group. Severe acute maternal morbidity in France: the EPIMOMS population-based study. Am J Obstet Gynecol. 2017;216:S345-S346.

16. Creanga AA, Berg CJ, Syverson C, Seed K, Bruce FC, Callaghan WM. Race, ethnicity, and nativity differentials in pregnancy-related mortality in the United States: 1993-2006. Obstet Gynecol. 2012;120:261-268.

17. Centers for Disease Control and Prevention. Severe Maternal Morbidity Indicators and Corresponding ICD Codes During Delivery Hospitalization. 2017. Disponible en: https://www.cdc.gov/reproductivehealth/ maternalinfanthealth/smm/ severe-morbidity-ICD.html.

18. American College of Obstetricians and Gynecologists and the Society for Maternal-Fetal Medicine, Kilpatrick SK, Ecker JL. Severe maternal morbidity: screening and review. Am J Obstet Gynecol. 2016;215:B17-B22.

19. Main EK, Abreo A, McNulty J, Gilbert W, McNally C, Poeltler D, et al. Measuring severe maternal morbidity: validation of potential measures. Am J Obstet Gynecol. 2016;214:643.e1-10.

20. Geller SE, Rosenberg D, Cox S, Brown M, Simonson L, Kilpatrick S. A scoring system identified near-miss maternal morbidity during pregnancy. J Clin Epidemiol. 2004;57:716-720.

21. You WB, Chandrasekaran S, Sullivan J, Grobman W. Validation of a scoring system to identify women with near-miss maternal morbidity. Am J Perinatol. 2013;30:21-24.

22. Pattinson R, Say L, Souza JP, Broek NV, Rooney C; WHO Working Group on Maternal Mortality and Morbidity Classifications. WHO maternal death and near-miss classifications. Bull World Health Organ. 2009;87:734.

23. Jakobsson M, Tapper AM, Palomäki O, Ojala K, Pallasmaa N, Ordén MR, et al. Neonatal outcomes after the obstetric near-miss events uterine rupture, abnormally invasive placenta and emergency peripartum hysterectomy - prospective data from the 2009-2011 Finnish NOSS study. Acta Obstet Gynecol Scand. 2015;94:1387-1394.

24. Adeoye IA, Onayade AA, Fatusi AO. Incidence, determinants and perinatal outcomes of near miss maternal morbidity in Ile-Ife Nigeria: a prospective case control study. BMC Pregnancy Childbirth. 2013;13:93. 